\title{
Molecular profiling of Adolescent Idiopathic Scoliosis: toward a comprehensive understanding of ais aetiology
}

\author{
Qing Yuan*, Nancy Karam, Lakshmi Suvarnan, Maryam Taheri, Bouziane Azeddine, DaShen Wang, \\ Sadallah Bouhanik, Guoruey Wong, Isabelle Turgeon, Benoit St-Jacques, Marie Yvonne Akoume Ndon, \\ Alain Moreau
}

From 7th International Conference on Conservative Management of Spinal Deformities Montreal, Canada. 20-22 May 2010

\section{Introduction}

The adolescent idiopathic scoliosis (AIS) is the most common deformity arising during childhood. Although its aetiology remains unknown, we have demonstrated a differential dysfunction of melatonin signalling through Gi proteins in osteoblasts isolated from AIS patients, leading to their stratification into three functional subgroups. Herein we examine the molecular profiles of these AIS groups using the functional classification developed by Moreau et al.

\section{Materials and methods}

Osteoblasts isolated from AIS patients $(n=3)$ were selected for each functional subgroup and compared to osteoblasts obtained from healthy controls $(n=3)$. In order to minimize variation among patients, we selected only female cases (mean age 14 years) having a severe double major curve. We used DNA chips with 54,000 probe sets that allow the analysis of the expression level of over 47,000 transcripts and variants from over 38,000 well-characterized human genes using the latest Gene Chip human genome array from Affymetrix (Human gene 1.0 ST). Processing of micro-array assays were performed at Genome Quebec Innovation Center in Montreal. Raw data were normalized using RMA (Robust Multi-array Analysis) method. Statistical analysis was performed by the EB (Wright and Simons) method using FlexArray software. Selection criteria for in-depth analysis include the magnitude of change in

Sainte-Justine Hospital, Montreal, Canada

Full list of author information is available at the end of the article expression (at least \pm 3 -fold) and $5 \%$ false discovery rate, which represent the highest stringency selection.

\section{Results}

Among 38,000 human genes tested, only 19 genes were shared and affected to the same extent in all AIS functional subgroups. We have found 8 genes specifically associated with the functional subgroup 1; 16 gene specifically associated with the functional subgroup 2 and finally, 11 genes specifically associated with the functional subgroup 3. Most of these genes encode for regulatory proteins such as transcription factors regulating axial skeleton and somite development, signalling molecules, and extracellular matrix proteins.

\section{Conclusion}

Our data further support our functional method of stratification of AIS patients and allowed the identification of novel genes associated with AIS. It is anticipated that identification of specific molecular markers will be used to identify children at-risk of developing scoliosis and to predict their clinical outcomes and response to novel pharmacological therapies.

Published: 10 September 2010

doi:10.1186/1748-7161-5-S1-016

Cite this article as: Yuan et al:: Molecular profiling of Adolescent Idiopathic Scoliosis: toward a comprehensive understanding of ais aetiology. Scoliosis 2010 5(Suppl 1):O16. 